\title{
EXTENDING AUTOMORPHISMS ON PRIMARY GROUPS
}

\author{
BY PAUL HILL
}

Communicated by George Seligman, June 10, 1968

Let $G$ be a $p$-primary abelian group, that is, let $G$ be a commutative group each element of which has finite order equal to a power of a fixed prime $p$. We suppose that $G$ is written additively and we say that an element $g \in G$ is divisible by a positive integer $n$ if there is a solution in $G$ to the equation $n x=g$. Note that 0 is divisible by every positive integer and that the collection of all the elements of $G$ with this property is a subgroup of $G$. This subgroup is denoted by $p^{\omega} G$. It is immediate that $p^{\omega}\left(G / p^{\omega} G\right)=0$. We can define $p^{\alpha} G$ for any ordinal $\alpha$ inductively by taking intersections at limit ordinals and letting $p^{\alpha+1} G=p\left(p^{\alpha} G\right)$.

It has been known for a long time that if the primary group $G$ is countable, then any automorphism of $p^{\omega} G$ can be extended to an automorphism of $G$; this result is implicitly contained in Zippin's proof [7] of Ulm's theorem. It is worth noting that if $G$ is countable, then $G / p^{\omega} G$ is a direct sum of cyclic groups according to a classical result due to Prüfer [6]: if $G$ is a countable primary group such that $p^{\omega} G=0$, then $G$ must be a direct sum of cyclic groups. This suggests the possibility of extending Zippin's result to the case where $G$ is any primary group with the property that $G / p^{\omega} G$ is a direct sum of cyclic groups. Recently, this result has been obtained by Hill and Megibben [3], [4], in a more general setting, and independently by Crawley [1]. What seems to the author as being a more striking generalization is the result being announced in this note, which says that the subgroup does not have to be $p^{\omega} G$ in order to extend height-preserving automorphisms.

By the height, $h_{G}(x)$, of an element $x$ in $G$ we mean the largest ordinal $\alpha$ such that $x \in p^{\alpha} G$ if such a largest ordinal $\alpha$ exists. Since $p^{\beta} G=\bigcap_{\alpha<\beta} p^{\alpha} G$ when $\beta$ is a limit, the only failure of the existence of such a largest ordinal is when $x \in p^{\alpha} G$ for all $\alpha$. In that case, we put $h_{G}(x)=\infty$ and adopt the convention that $\infty>\alpha$ for all ordinals $\alpha$. If $\pi$ is an automorphism of a subgroup $H$ of $G$, we say that $\pi$ is heightpreserving in $G$ if $h_{G}(x)=h_{G}(\pi(x))$ for all $x$ in $H$. Denote by $Q^{G}(H)$ the group of automorphisms of $H$ that preserve heights in $G$ and let $a_{H}(G)$ denote the group of automorphisms of $G$ that map $H$ onto $H$. Naturally, we write $Q(G)$ for $Q^{G}(G)$ since the latter is the full automorphism group of $G$, and we write $Q(G)$ instead of $a_{0}(G)$ for the 
same reason. Observe, more generally, that $Q(G)=\mathfrak{Q}_{H}(G)$ for any invariant subgroup of $G$; in particular, $Q(G)=Q_{p \omega G}(G)$. On the other hand, if $\pi \in Q_{H}(G)$ for any subgroup $H$ of $G$, then the restriction of $\pi$ to $H$ is necessarily in $Q^{G}(H)$. Thus there is a natural map: $Q_{H}(G)$ $\rightarrow a^{G}(H)$.

Theorem. Suppose that $G$ is a primary abelian group and that $H$ is a subgroup of $G$ such that $G / H$ is a direct sum of cyclic groups. Then the natural map $\mathfrak{Q}_{H}(G) \rightarrow Q^{G}(H)$ is epic, that is, any height-preserving automorphism of $H$ can be extended to an automorphism of $G$.

The proof will appear elsewhere [2]. Since $a_{p^{\omega} G}(G)=Q(G)$ and since $Q^{G}\left(p^{\omega} G\right)=Q\left(p^{\omega} G\right)$, we have

Corollary (Hill and Megibben [3], Crawley [1]). If $G / p^{\omega} G$ is a direct sum of cyclic groups, then $a(G) \rightarrow Q\left(p^{\omega} G\right)$ is epic.

The next result is related to [5].

COROLlaRy. If $L$ is a large subgroup of the primary group $G$, then the natural map $Q(G)=Q_{L}(G) \rightarrow Q^{G}(L)$ is epic.

\section{REFERENCES}

1. P. Crawley, Abelian p-groups determined by their Ulm sequences, Pacific J. Math. 22 (1967), 235-239.

2. P. Hill, Endomorphism rings generated by units, (to appear).

3. P. Hill and C. Megibben, Extending automorphisms and lifting decompositions in abelian groups, Math. Ann. 175 (1968), 159-168.

4. - Direct sums of countable groups and generalizations, Proc. of the Montpellier Conference, (to appear).

5. C. Megibben, Large subgroups and small homomorphisms, Michigan Math. J. 13 (1966), 153-160.

6. H. Prüfer, Unendliche abelsche Gruppen von Elementen endlicher Ordnung, Dissertation, Berlin, 1921.

7. L. Zippin, Countable torsion groups, Ann. of Math. 36 (1935), 86-99.

UNIVERSITY OF HoustoN 